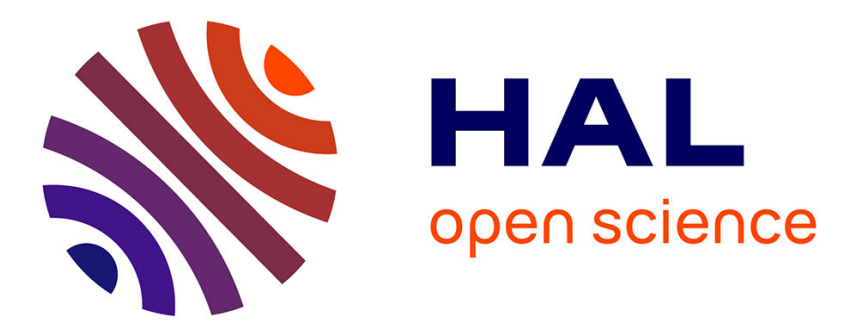

\title{
La viralité entre métaphore communicationnelle et approche esthétique
}

Pierre Morelli

\section{To cite this version:}

Pierre Morelli. La viralité entre métaphore communicationnelle et approche esthétique. Colloque international L'image à l'épreuve de la viralité, Institut Supérieur des Arts et Métiers de Mahdia (Tunisie), Nov 2016, Mahdia, Tunisie. pp.273-294. hal-01721601

\section{HAL Id: hal-01721601 \\ https://hal.univ-lorraine.fr/hal-01721601}

Submitted on 24 Jun 2018

HAL is a multi-disciplinary open access archive for the deposit and dissemination of scientific research documents, whether they are published or not. The documents may come from teaching and research institutions in France or abroad, or from public or private research centers.
L'archive ouverte pluridisciplinaire HAL, est destinée au dépôt et à la diffusion de documents scientifiques de niveau recherche, publiés ou non, émanant des établissements d'enseignement et de recherche français ou étrangers, des laboratoires publics ou privés. 


\section{Pierre MORELLI}

«La viralité entre métaphore communicationnelle et approche esthétique » in : Dialogue des révolutions : LA VIRALITÉ

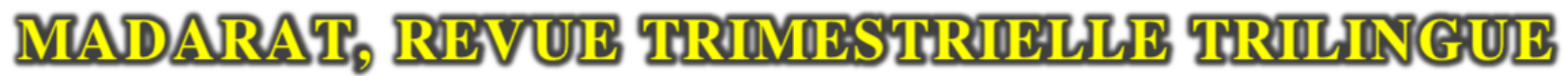 MULTODISCIOPLINAVRE}

N²9-30 : été - printemps 2017

Association Madarat Maarifia

BP. 243 La manouba 2010 Tunisie

associamadarat@gmail.com

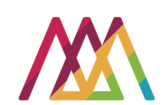

ISAMM Actes du colloque international : L'image à l'épreuve de la viralité. Organisé par l'Institut Supérieur des Arts et Métiers de Mahdia (Tunisie) les 18-19-20 novembre 2016.

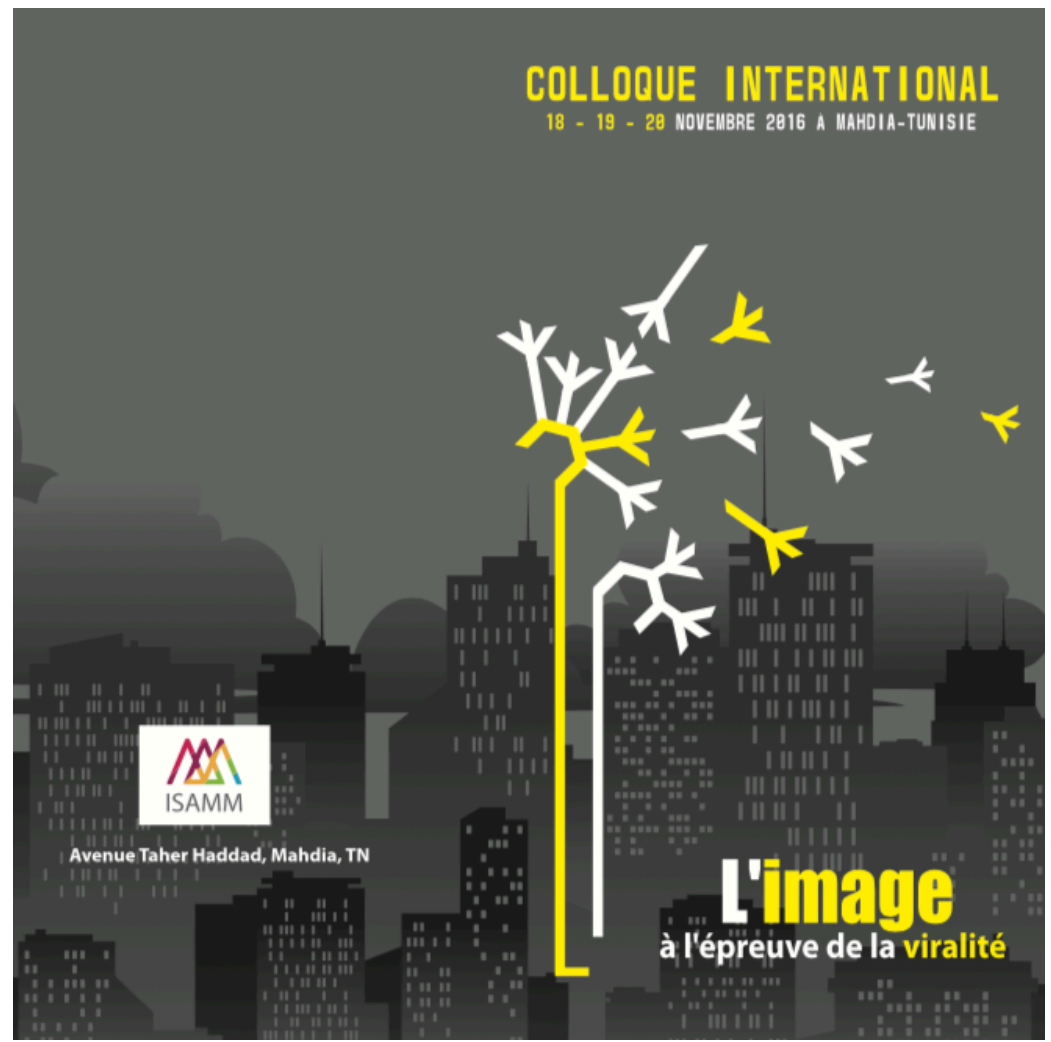

Pour citer ce chapitre :

Morelli P. (2017). La viralité entre métaphore communicationnelle et approche esthétique, Madarat, $\mathrm{n}^{\circ} 29-30$. Dialogue des révolutions : la viralité, 273-294.

Ce document est mis à disposition selon les termes de la licence Creative Communs : attribution, pas d'utilisation commerciale, pas de modifications 3.0.

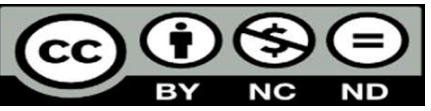




\title{
LA VIRALITÉ ENTRE MÉTAPHORE COMMUNICATIONNELLE ET APPROCHE ESTHÉTIQUE
}

\author{
Pierre MORELLI
}

\author{
Maître de conférences \\ Centre de recherche sur les médiations \\ Université de Lorraine \\ pierre.morelli@univ-lorraine.fr \\ http://crem.univ-lorraine.fr/morelli-pierre
}

Près de vingt ans après l'entrée dans la société de l'information', les effets croisés de la mise au point d'interfaces d'écritures simplifiées ${ }^{2}$ par les acteurs du web 2.0 et de la montée en puissance de la culture de l'usage des technologies de l'information et de la communication (TIC) ont favorisé la démultiplication des pratiques numériques. Conjuguant l'intervention de spécialistes avec celle de profanes, l'information en ligne opère désormais selon un mode conversationnel (Granier, 2001) dont les médias traditionnels (presse écrite, radio et télévision) ont vite saisi la portée, cherchant à composer avec les réseaux socionumériques ${ }^{3}$ afin d'étoffer le lien avec leurs publics jusqu'à être parfois débordés par l'extraordinaire réactivité des internautes. Si Facebook et Twitter permettent aux médias faire écho en ligne à leurs programmes, leurs succès d'estime confèrent à ces espaces de médiation et de conversation un statut de média à part entière avec lequel les médias doivent sinon rivaliser, tout au moins composer. Contre la position de surplomb des médias de masse s'installent alors des circulations d'information transversales auxquelles tout un chacun peut tenter de prendre part. Snapchat et Instagram vont plus loin que Facebook et Twitter. Prenant acte du développement de l'internet mobile, principalement destinés à l'échange photographique, ces deux réseaux sociaux accordent désormais à l'image une place centrale dans les conversations numériques dont l'un des objectifs consiste à créer le «buzz » afin de capter en le focalisant l'intérêt des internautes. Notre rapport à l'image ayant changé avec le développement de l'internet, cette dernière se retrouve en effet au cœur de processus médiatiques qui, en incrémentant le nombre de vues, stimulent sa visibilité sur la toile (Gunthert, 2009).

\section{L’image au service de la créativité des internautes}

L'avènement du web 2.0 , et plus précisément le développement de la participation active des internautes à l'écriture en ligne, favorise l'expression de la créativité de quidams internautes et bénéficie de leur capacité à s'approprier les artefacts à des fins purement personnelles ou marketing. Rappelons l'histoire de Clever Da Silva (De Kerkhove, 1997 : 255). Amoureux de sa ville et internaute éclairé, ce chauffeur de taxi new-yorkais va très vite réaliser l'intérêt marketing que peut alors représenter le web. S'attachant les services d'un designer (Glen de Vries) et d'un producteur de sites web (Richard Oceguera) il entreprend de créer, en 1999, un site web invitant les internautes à suivre les trajets de son véhicule et à correspondre avec lui par e-mail ${ }^{4}$. Cette caméra itinérante amenait les internautes à découvrir la ville de New York à

\footnotetext{
${ }^{1}$ La France décrète en 1998 le «Plan d'action gouvernemental pour la société de l'information ». L'enjeu de ce projet global était de rattraper le retard par rapport aux pratiques nord-américaines en matière d'usage des TIC et de prendre la mesure des importantes évolutions contemporaines (sociales, politiques, économiques, législatives...) de sorte à développer, à cours et moyen terme, à travers des initiatives interministérielles, l'usage des TIC.

${ }^{2}$ Les CMS (Content Management Services) permettent au tout venant d'écrire sur la toile tant pour exprimer une vision, un point de vue que pour commenter, discuter et débattre des propositions mises en ligne par d'autres personnes, sur les sites web et à travers les murs des réseaux sociaux.

${ }^{3}$ Rappelons les principaux réseaux sociaux numériques: Blogs, Myspace puis Facebook, Flickr, Linkedin, Twitter et enfin Spotify, Tumblr, Foursquare, Google+, Pinterest, Snapchat...

${ }^{4}$ Les photographies mises en ligne étaient renouvelées deux fois par minute du lundi au vendredi entre $9 \mathrm{~h}$ et $18 \mathrm{~h}$, heure locale.
} 
partir du «point de vue »s localisé dans le véhicule en mouvement dans les rues de New York. Cette visite virtuelle préfigure, remarquons-le deux types de dispositifs dont l'internaute peut disposer de nos jours. Il s'agit tout d'abord des milliers de webcams qui quadrillent l'espace publique des villes selon un maillage prédéterminé et qui, par une diffusion en direct d'images vidéos ou de photographies rafraîchies toutes les « $\mathrm{x}$ » secondes, offrent à l'internaute une vision panoptique de quartiers choisis. Le site web de Clever Da Silva s'apparente également aux «street views», assemblages photographiques constitués de continuum de prises de vues fixes proposés par les grands moteurs de recherche et enrichis par toutes sortes d'informations pratiques et/ou marketing, outils forts utiles pour anticiper de futurs trajets urbains. Entre déplacements réels et mises en circulation de données, les réseaux numériques véhiculent donc différents types d'informations intelligibles et interprétables que Google Map ou Bing , par exemple, associent à leurs cartes géographiques. Une fois mises en forme, ces données véhiculées par les différents réseaux (internet, GPS') sont intégrées dans les représentations mises en ligne (cartes...) parfois même pour symboliser les flux de circulations.

Or cette créativité qui se développe désormais à l'aune de l'intensification de conversations numériques procède, selon Yves Jeanneret, par la mise en circulation et le partage massif d'informations sur la toile. Ce chercheur choisit de nommer le processus « trivialité » non pas pour désigner la dimension vulgaire ou banale que peuvent prendre les échanges mais en référence au latin, trivium signifiant carrefour. Pour Yves Jeanneret (2008 : 13) les idées et les objets qui circulent sur l'Internet constituent autant d'éléments susceptibles d'être repris en masse et déclinés donc transmis et métamorphosés ce qui les rend susceptibles de produire du nouveau voire à se charger de valeur. En effet, précise-t-il : « Les hommes créent, pérennisent et partagent des êtres culturels qu'ils élaborent en travaillant les formes que ces derniers peuvent prendre et en définissant la façon dont ces formes font sens » (Ibid.). Par être culturel cet auteur désigne «un complexe qui associe des objets matériels, des textes, des représentations et qui aboutit à l'élaboration et au partage d'idées, d'informations, de savoirs, de jugements » (Ibid.: 16). Rappelant que la culture est faite de la reprise et de la reconstruction constante des objets et de leurs formes et que les hommes ont besoin pour élaborer leur culture de travailler la matière, de concevoir des techniques, de façonner des objets et qu'à chaque appropriation des objets, Yves Jeanneret affirme que l'histoire des investissements, ouvre de nouveaux espaces symboliques, porteurs de sens et de liens (Ibid.: 17), ce cheminement à travers les carrefours de la vie sociale (Ibid. : 14) s'établissant selon un processus social original comprenant de la transmission, de la traduction, de l'interprétation, de la tradition.

\section{Le réseau à l'épreuve de l'avènement des conversations numériques}

Avec le développement exponentiel de l'usage et la généralisation de la connectivité qu'apportent et cultivent l'internet mobile et l'internet des objets les réseaux participent de la vie quotidienne. Il deviennent à la fois des modèles fonctionnels de la complexité contemporaine dont ils contribuent à faire comprendre la portée et des prothèses perceptives indispensables pour participer aux échanges. Articulant formes hypertextuelles et modalités dialogiques, le web 2.0 constitue ainsi un terreau riche en expression de la créativité de l'internaute et en formes communicationnelles nouvelles caractérisées par la réciprocité des échanges, et l'influence croisée des paradigmes de la co-construction et de l'extémité de l'information (Tisseron, 2011).

\footnotetext{
${ }^{5}$ «Prenez place dans mon taxi virtuel et permettez-moi de vous montrer la ville de New York à partir de mon point de vue » : "So take a seat in my Internet Cab and let me show you New York City from my point of view". $\mathrm{Nb}$ : le site http://www.ny-taxi.com/ n'est plus accessible, quelques pages sont néanmoins consultables sur: https://web.archive.org/web/20020205111623/http://www.ny-taxi.com/cabcam/live.asp.

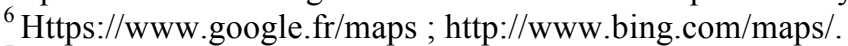

${ }^{7}$ Rappelons que les GPS de voiture signalent également les travaux et ralentissements présents sur le trajet choisi.

${ }^{8}$ Google et Bing proposent d'afficher des informations concernant le trafic automobile selon un code de couleur : rouge pour trafic arrêté ou fortement ralenti, vert pour trafic fluide.
} 
Acteurs et/ou observateurs de l'activité menée au sein des réseaux, des communautés d'internautes investissent les dispositifs d'interconnexion proposés par le web selon des modalités d'échanges mêlant fluidité de l'oral et capacités d'archivage et d'organisation (De Kerkhove 1999: 85). Le fonctionnement même du réseau' apporte aux traces d'action textuelles et iconographiques, qui relèvent plus traditionnellement de l'écrit, la souplesse discursive de l'oral. Â chaque instant réactivé, cet alliage d'oralité et d'écriture construit une mise en mémoire commune et partagée des pensées et des activités humaines. Interactive, cette construction s'établit selon un mode dialogique articulant «informations codées et traitées par calcul automatique entre l'homme et l'ordinateur» (Couchot, 2007: 199). Coexistent et s'entrecroisent alors paroles profanes et paroles savantes au risque de produire des scories communicationnelles, source d'interrogation dont s'emparent volontiers certains artistes $^{10}$. Or, fidèle au principe de la libre circulation des idées inscrit dans les gènes de l'Internet (Breton, 2000: 57), la propagation de l'information à travers ces réseaux opère essentiellement de manière virale. La métaphore de la viralité semble d'emblée fort bien adaptée à la modélisation des phénomènes de circulation de contenus informationnels via les réseaux sociaux. Reprises, décontextualisées et recontextualisées selon le bon vouloir des internautes les propositions circulent en effet avec des vitesses vertigineuses, favorisant même le dépassement des frontières politiques et linguistiques.

Si la viralité informationnelle peut être pensée comme mécanisme de propagation au sein d'une unité se comportant comme une entité biologique victime d'une attaque virale (infection, défenses naturelles...), la métaphore suffit-elle pour autant à modéliser les mécanismes communicationnels en vigueur sur la toile ? Proposons de tenter ici d'aller audelà (ou en deça) de la métaphore. Commençons donc par interroger, par exemple, les modalités mêmes de transmission de «cellule à cellule » ainsi que la décision propre à chaque récepteur de choisir entre quatre positions à adopter: ignorer l'information, la relayer telle quelle, s'appuyer sur sa force illocutoire afin de formuler un message à forte portée perlocutoire et donc la détourner à son profit ou engager une procédure pour en minimiser la portée ou en condamner la fausseté et en dénoncer l'incohérence. La circulation virale de l'information suppose alors que l'action des internautes ne se limite pas au seul rôle de relai d'information (Retweet) ou de marquage par approbation (Like). Pour intéressant qu'il semble être, le modèle fonctionnel de la viralité ne suffit cependant pas à circonscrire les enjeux communicationnels de la contamination informationnelle. Ouvrons la boite noire" afin et de discriminer le cadre de fonctionnement ${ }^{12}$ et le cadre d'usage ${ }^{13}$ de ce modèle. Cette déconstruction s'accompagnera de la mise en tension d'une analyse communicationnelle et d'une approche esthétique.

\section{Dispositions communicationnelles de la viralité}

$\mathrm{Si}$, penser la propagation de l'information à partir du principe de viralité permet de questionner les «phénomènes de circulation des contenus informationnels en mettant l'accent sur l'intrication spécifique au web des logiques communicationnelles et des logiques d'audience » (Beauvisage et al., 2011 : 152), il convient d'aller au delà de l'immédiateté de la métaphore. La viralité gagne en effet à être analysée en profondeur afin de comprendre les dispositions communicationnelles qui l'animent. Pour Thomas Beauvisage, Jean-Samuel Beuscart, Thomas Couronné et Kevin Mellet (op. cit. : 153-158) la viralité peut à la fois se définir comme forme de concentration temporelle de l'attention sur un contenu, comme

\footnotetext{
${ }^{9}$ Recopie dans des sites miroirs, reprise partielle des messages utilisées dans la rédaction des courriels voire « retweets », gels d'écrans et propension à faire circuler donc à rendre visibles les informations...

${ }^{10} \mathrm{~L}$ 'artiste Carl Y. voit dans la création sur internet, qu'il juge florissante, l'apparition d'une « culture du déchet, voire de poubelle » : Mcd (Musiques \&cultures digitales), nº69, décembre-janvier-février 2013, p. 30.

${ }^{11}$ Nous référons ici aux travaux de Patrice Flichy (1995).

${ }^{12}$ À quels mécanismes créatifs et communicationnels l'exercice de la viralité fait-il appel ? Quels sont la place et le rôle du traitement informatique de l'information dans la transmission virale de l'information?

${ }^{13}$ Comment s'applique le principe de viralité dans la transmission des informations.
} 
mécanisme de circulation desdits contenus ${ }^{14}$ et enfin comme «contagiosité », à savoir l'aptitude du réseau à influencer la diffusion de certains contenus doublée d'un besoin d'expliciter les principes de contagion inter individuelle. Cette triple visée s'articule, pouvons nous l'observer, autour d'un élément central : le contenu. Objet de la communication ou catalyseur communicationnel, le contenu peut avoir une valeur informative, symbolique ou purement émotionnelle. La dimension émotionnelle constitue le moteur principal de la contagion d'information. Ce n'est pas un fait nouveau. La recherche de l'émotion est, rappelons le, parfaitement maitrisée par les grands médias dans la conception de leurs programmes.

Deux exemples récents illustrent la vitesse avec laquelle la toile s'empare d'informations à forte charge émotionnelle et qui trouvent un important écho sur la toile indépendamment de la langue, de la culture et de la situation géographique des internautes. L'adoption rapide, massive du hashtag «\#JeSuisCharlie » (cf. Figure 1) proposé et massivement diffusé peu de temps après l'annonce de l'attentat visant les locaux du journal satyrique Charlie hebdo à Paris, le 7 janvier 2015 a provoqué une réaction planétaire dont la rapidité et l'ampleur peuvent être visualisées de manière graphique's. Touchés par la brutalité de l'information, les internautes ont massivement relayé cet hashtag, formant une réaction en chaine qui connut un point culminant à 21 h 30 (heure de Paris) avec 6500 tweets par minute.
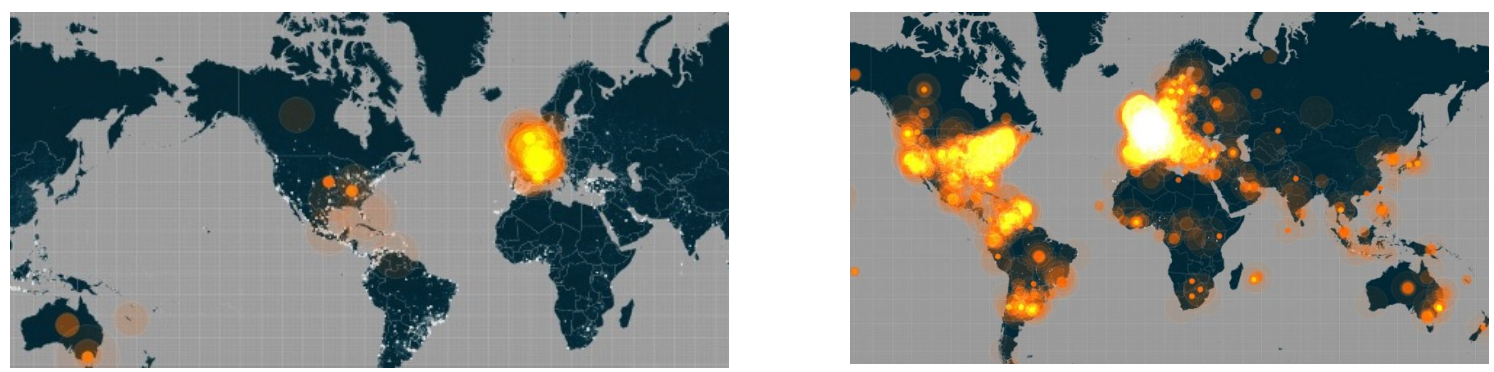

Figure 1: Mercredi 7 janvier 2015. Deux images qui permettent de visualiser la propagation du hashtag (\#JeSuisCharlie) à travers le monde durant les premières 24 heures.

Compte-tenu des contraintes d'écritures imposées par Twitter, les courts messages et les redirections concentraient le propos autour de l'hashtag « \#JeSuisCharlie » qui, à mesure que grandissait le phénomène gagnait en retour en valeur symbolique et émotionnelle. L'effet de contamination de l'information est ici aussi fulgurant que planétaire.

Par sa puissance évocatrice, l'image photographique est également capable d'embraser la toile à force de reprises ${ }^{16}$ et de commentaires postés via les réseaux sociaux. Rappelons nous la photographie d'Alan Kurdi, enfant syrien d'origine kurde ayant fuit avec sa famille la ville de Kobané assiégée. Ce bambin de 3 ans fut retrouvé mort-noyé, face contre terre, comme une poupée rejetée par les flots sur une plage de Turquie le 2 septembre $2015^{17}$. Donnant au drame collectif une dimension singulière et poignante, le cliché s'est rapidement retrouvée au cœur d'intenses échanges sur la toile et fut abondamment repris par les médias d'information du monde entier. Devenue iconique, l'image de ce petit corps d'enfant rejeté par les flots sur la plage a largement été transmise (et retransmise), déclinée et traduite (retranscrite) sous forme

\footnotetext{
${ }^{14}$ Mécanisme qui selon Beauvisage et al. relève soit de la circulation à proprement parler (ces auteurs parlent alors de « contagion sociale au sens strict») soit d'une éditorialisation propre aux dispositifs que sont les réseaux socionumériques.

${ }^{15}$ Voir la carte animée : https://srogers.carto.com/viz/123be814-96bb-11e4-aec1-0e9d821ea90d/public_map.

${ }^{16}$ Messages relayés tels quels ou compositions qui déclinent avec une empathie généralisé le sentiment de révolte et d'incompréhension partagé par les internautes.

${ }^{17}$ En France, ce drame est arrivé le lendemain de la rentrée scolaire. On peut imaginer combien cette image dénotait par rapport à l'ensemble des reportages et sujets traditionnellement proposés en cette occasion et qui mettent en scène les enjeux de l'école, donc l'avenir des enfant et le ressentis d'élèves à l'aube d'une nouvelle année scolaire qui commence...
} 
de dessins et autres montages photographiques dénonçant l'impuissance contemporaine voire une certaine lâcheté face au drame humanitaire qui se joue en Méditerranée donnant corps à cette « formule» particulière que propose la trivialité : les mèmes ${ }^{18}$.

Observée comme ressort communicationnel cette propension à décliner un même thème intervient de manière virale. Si elle peut être modélisée par l'empathie, propre à la dimension médiatique, l'environnement informatique qui englobe la construction et la diffusion de l'information correspond à la mise en œuvre de la créativité variationnelle (Moles, 1990), caractéristique intrinsèque à la création par ordinateur consistant à faire varier des modèles. L'exploration des changements possibles de formes précédentes, la déformation d'une forme ou de ses parties ou de ses caractéristiques (contraste, couleurs, point de vue...) peut être alors menée selon des tâtonnements créatifs sans avoir à rendre compte des écarts provisoires ou au contraire contribuer, pour peu qu'un large consensus se dégage à produire du sens auquel les internautes adhèrent à force de commentaires et de reprises et qu'ils contribuent à faire connaître. Remarquons que cette production de sens s'inscrit dans ce que Yves Jeanneret (2008 : 87) désigne par « altération », à savoir « le processus qui veut qu'en se déplaçant dans la société les idées et les textes ne cessent de se transformer » et qu'elle renforce à mesure que sa visibilité augmente et s'affirme en ligne.

\section{Les mèmes numériques: fruit de la créativité variationnelle et vecteurs communicationnels}

Qu'elle soit, selon la différenciation proposée par la Judith Schlanger (1983), fertile ou féconde', la créativité sur l'internet s'inscrit dans un élan culturel. Or, avec le développement de l'internet, l'accès en temps réel à des banques de données textuelles et iconographiques favorise la prise d'images $\mathrm{du} \mathrm{web}^{20}$, faciles à retraiter ${ }^{21}$ et à décontextualiser pour les recontextualiser à dessein. Bénéficiant des potentialités variationnelles offertes par l'ordinateur, les internautes s'expriment et communiquent à travers l'élaboration et la mise en circulation de «mèmes numériques » (Bonenfant, 2014). Phénomène viral et souvent banal qui se démarque du flot continu de la production d'informations mises en lignes chaque minute sur l'internet (ibid.: 29), le «mème numérique » peut présenter différentes formes sémiotiques (images fixes ou mobiles, textes, sons...) et viser des objectifs humoristiques, commerciaux, politiques ou esthétiques. Signe d'une culture partagée qui s'affiche et se tisse à force de productions caractéristiques propres aux mondes en ligne, le "mème numérique » agit sur la communication et produit un effet structurant pour les communautés en ligne (ibid.: 28). Traces de productions culturelles s'exerçant sur la toile, objets et facteurs de consolidation de l'appartenance à une communauté émotionnelle ou d'intérêt (Morelli, 2016), et entités à forte charge culturelle assujetties à reprise et à métamorphose (Jeanneret, 2008), les «mèmes numériques » se renforcent sous l'effet des réseaux qui ne cessent de quantifier les phénomènes qui les traversent, ce qui en retour ajoute en visibilité.

Deux types de diffusion des mèmes peuvent être identifiés précise Maud Bonenfant: la réplication et la variation. Si dans la réplication le matériel viral circule à l'identique, l'internaute peut aller au delà de pareil rôle de relai d'information et engager " un certain nombre d'altérations changeant «légèrement ou drastiquement le sens du matériel

\footnotetext{
${ }^{18}$ Pour Yves Jeanneret, la « conscience de plus en plus vive de l'importance de la trivialité a donné du succès à des formules comme le buzz, la viralité, les mèmes sur Internet » (cf. : http://effeuillage-la-revue.fr/portfolioitem/la-trivialite-par-yves-jeanneret/, brève présentation du concept de "trivialité disponible en ligne. Consulté le 19 décembre 2016. Le buzz correspond à l'effet, la dimension quantitative rapporté à un moment, à une temporalité de la circulation d'informations, la viralité est prise ici comme modalité(s) multidirectionnelle(s) de circulation des informations et phénomènes de contamination alors que la notion de même désigne les éléments propagés et déclinés à travers le web (image, hashtag, phrase ou mot, personnage).

${ }^{19}$ L'invention fertile est d'ordre quantitatif (la qualité des propositions importe peu) alors que l'invention féconde peut au contraire engager du nouveau (changement paradigmatique ou émergence de nouveaux sujets de discussion).

${ }^{20}$ Par téléchargement ou copie d'écran.

${ }^{21}$ Par copié/collé, recadrage, modification chromatique...
} 
"d'origine" » (ibid.: 29). Volontaires ou fortuites, ces altérations procèdent de la même logique que suit la créativité variationnelle. Cette différenciation n'est toutefois pas aussi nette ou définitive qu'elle puisse paraître car, saisie par la technologie et fruit de l'action humaine, la réplication a de fortes chances d'être elle-même assujettie à variations.

La copie de soi et la réplication sont, en effet, des principes bien connus par la génétique. Parmi l'ensemble des composés organiques, affirme l'ethnologue Richard Dawins (1996: 35), il existe une molécule remarquable possédant «l'extraordinaire pouvoir de pouvoir créer des copies d'elle-même ». C'est un « replicateur » qui se présente selon une chaine complexe de différents éléments constitutifs selon des séquences capables de copier « le réplicateur luimême » (ibid.). Fait notable, les répliques ne sont pas forcément identiques, la copie du réplicateur pouvant introduire d'infimes erreurs dommageables ou bénéfiques à la survie du modèle. Dans le premier cas l'erreur provoque une divergence qui, à long terme peut s'essouffler d'elle même, faute d'être recopiée en tant que telle ou d'aboutir à un élément qui s'écarte définitivement de la proposition originelle. Dans le second cas, la différence introduite par l'erreur participe, insiste Richard Dawkins, de la progressivité même qui saisit l'évolution génétique. L'écart est alors fécond.

Remarquons toutefois que la contagion informationnelle «ne se suffit pas à elle seule. Elle s'articule avec des actions d'éditorialisation » (Beauvisage et al., $2011: 157$ ) qui, à travers les réseaux sociaux, sont en partie déléguées à l'internaute. Depuis l'avènement du web 2.0, l'éditorialisation résulte aussi de la recommandation sociale (Tags, Like) donc du marquage des contenus par l'internaute (ibid.: 156). Opérant de proche en proche, la transmission procède par enrôlement. Chaque destinataire peut en effet devenir à son tour destinateur et relai amplificateur de la transmission du fait des potentialités relationnelles que représentent le partage de murs d'information (Facebook) ou la redirection à l'ensemble des abonnés (Twitter, Linkedin,...). Qu'il soit relayé tel quel (retweet), annoté («liké », commenté ou repris donc recontextualisé au bon vouloir du destinateur le message assume aussi bien une fonction phatique ${ }^{22}$, conative ${ }^{23}$ et référentielle voire méta-linguistique ${ }^{24}$ (Jakobson, 1963).

Bénéficiant de la propension des réseaux à recycler les contenus, et profitant de l'essor que connaît, depuis le web 2.0 l'appropriation sociale des technologies ${ }^{25}$ la circulation d'information s'établit désormais selon des formes conversationnelles (Granier, op. cit) ce qui rajoute en visibilité. Générées par l'image et le texte au sein des réseaux socionumériques contemporains, les conversations numériques participent en effet au renforcement de contamination informationnelle à travers la démultiplication de «l'infection » notamment par création de variétés différentes de répliques (Dawkins, 1996: 36-37) et via le processus d'enrôlement d'individus devenus par la suite agents réplicateurs. Opérant sur le principe de la créativité variationnelle, le «mème numérique» nous amène, d'un point de vue communicationnel à prolonger la métaphore virale par une métonymie. Le destinataire d'une information devient alors potentiellement destinateur. Ce n'est pas seulement le message qui se transmet à travers la toile c'est aussi un processus d'enrôlement amenant l'internaute à devenir relai d'information ou agent réplicateur soucieux d'en raviver l'intérêt à l'intérieur de ses communautés ${ }^{26} \mathrm{~d}^{\prime}$ 'appartenance, faute de quoi la propagation virale de l'information en ligne risquerait de s'épuiser d'elle même.

\footnotetext{
${ }^{22}$ Vérification du fonctionnement des liens au sein du réseau.

${ }^{23}$ Interpeller les membres de son réseau permet également de se rappeler à leur bon souvenir, à asseoir une image dynamique donc à s'y distinguer par son activité.

${ }^{24}$ Dimensions culturelles qui unissent les membres d'un même réseau.

${ }^{25}$ Et notamment aux questions de détournement et de braconnage (De Certeau, 1980) et de l'appropriation des TIC (Jouet, 2000 ; Proulx, 2005). Remarquons en outre que la dimension sociale de l'appropriation intervient également dans le champ de l'art ( $c f$. «L'œuvre d'art à l'ère de son appropriabilité numérique », Gunthert, 2011). ${ }^{26}$ Chaque internaute est en effet membre de plusieurs communautés qui échangent en ligne. Ce qui ajoute aux potentialités de diffusion de l'information.
} 
Dialoguer, collaborer et créer à distance se construit progressivement à mesure que chacun apprivoise les outils et les environnements donc les réseaux numériques. La montée en puissance de la pratique s'effectue toutefois dans un cadre de perception fortement contraint par le caractère immersif et invasif des technologies numériques, ce qui oriente et limite chez l'usager l'observation et le jugement. À ce titre, la viralité comporte également une dimension esthétique.

\section{Réseaux et perceptions contemporaines}

Sous l'impulsion des technologies contemporaines, la réalité change, et cette "réalité en mutation », insiste Paul Virilio (1988: 14), ne peut plus être représentée avec les outils traditionnels car:

$$
\begin{aligned}
& \text { «...le monde ne pourra plus se représenter désormais à travers une sculpture, ou l'image fixe du } \\
& \text { tableau, il ne pourra trouver sa juste "représentation" que dans la vitesse du mouvement, la } \\
& \text { juxtaposition des sources d'informations, l'hétérogénéité simultanée de ses supports physiques et } \\
& \text { électroniques ». }
\end{aligned}
$$

C'est pour cela que nous considérerons volontiers les réseaux comme appareils au service d'une vision renouvelée des rapports sociaux contemporains à leur développement. Les technologies, précise Jean-Louis Déotte (2004: 106 et 109), déterminent le mode même d'apparition des événements et inventent des temporalités et des spatialités propres à chaque époque. À travers les technologies s'élaborent des représentations qui déconstruisent les relations spatio-temporelles tant sur le plan formel qu'en profondeur.

L'appareil dont il est question ici, dirons-nous en référence à Pierre-Damien Huygues (2005 : $25)$, n'est toutefois pas un instrument au sens pratique du terme. Il met au point un état possible de la «corrélation» entre «un sujet et un objet», opérant au service de la perceptibilité ${ }^{27}$. Tout comme l'invention de la perspective au Quatrocento a marqué un renouveau de la représentation picturale (Déotte, 2001) la photographie, le cinéma et, à la charnière du $\mathrm{XX}^{\circ}$ et $\mathrm{XXI}^{\circ}$ siècle, les réseaux ouvrent chacun une nouvelle époque.

Les réseaux constituent donc des appareils qui affectent la perception du monde contemporain et participent de la compréhension et de la modélisation des réalités que les technologies et l'usage des technologies construisent. L'art engage ce type d'approche. À force d'explorations plastiques et esthétiques les artistes contribuent à modéliser et à analyser, la complexité communicationnelle contemporaine. Leur regard distancié propose une position de surplomb et offre un recul critique bénéfique à l'interrogation des enjeux sociaux et sociétaux contemporains produits et d'une certaine façon révélés par les réseaux numériques. Spectateurs et acteurs du développement des technologies numériques ils assument également un rôle exploratoire et critique des systèmes techniques qu'ils digèrent et tordent à force de traitements plastiques et de propositions esthétiques. Leurs interventions artistiques au sein des réseaux numériques ne visent pas forcément à utiliser les dernières technologies disponibles, même si les approches s'affinent et se reconfigurent au gré de l'évolution technologique. Elles contribuent à la production de formes symboliques qu'usagers et acteurs des réseaux s'approprient, banalisent et utilisent souvent même sans en interroger les origines. Questionnés par l'art, peuplés de nombreuses propositions artistiques qui les bousculent, les réseaux socio-numériques constituent en fait de véritables appareils de perception et d'analyse de la complexité communicationnelle contemporaine. Les modélisations qu'ils proposent aident alors à comprendre, par l'usage et par l'expérience esthétique comment se construisent et comment se croisent désormais les échanges interpersonnels.

\footnotetext{
${ }^{27}$ D’un point de vue étymologique, précise Jean-Louis Déotte (2004 : 99), appareil vient du latin apparare qui signifie « préparer pour, préparatif » de manière «à respecter la loi de l'accueil de l'événement» de "penser le paraître comme un fait » rajoute Pierre-Damien Huyges (op.cit. : 24).
} 
Visionnaires, à force d'expérimentations plastiques et de recherches esthétiques menées à travers des installations interactives, les artistes redoublent, via un ensemble d'expérimentations plastiques, les interrogations scientifiques contemporaines ${ }^{28}$ au profit de questions sociales et sociétales (Morelli, 2000). Ils saisissent les réseaux autant comme lieux et sièges de la propagation de l'information, donc comme modèles fonctionnels de la transmission virale de l'information dont ils contribuent à faire comprendre la portée que comme prothèses perceptives indispensables à la compréhension de la complexité contemporaine. La viralité peut alors être pensée comme moteur esthétique.

\section{Vivre, expérimenter et questionner les phénomènes viraux}

Conçu en 1986 et mis en œuvre dès 1987 via le réseau minitel, Le générateur poḯtique (Figure 2) ${ }^{29}$ d'Olivier Auber est un dispositif créatif interactif ${ }^{30}$ fondé sur le principe des cadavres exquis surréalistes. Il permet d'offrir l'expérience d'une création collective à des quidams pouvant visualiser en temps réel les propositions des autres participants. Il donne vie à des moments de création partagée, pendant lesquels chacun peut influencer l'autre ou lui répondre alors que s'instaurent des complicités entre les actants. Ce dispositif permet en outre d'observer, de provoquer un sentiment de panique face aux propositions environnantes. Il sera perfectionné passant de la technologie du minitel à des versions IP conçues pour l'Internet $(1995,1997)$ puis des versions mobiles en 2012 et, en 2015, une version ${ }^{31}$ hybridant dispositif mobile et image résultante projetée sur le sol, invitant chacun à occuper l'espace correspondant au dessin qu'il dessine en temps réel.

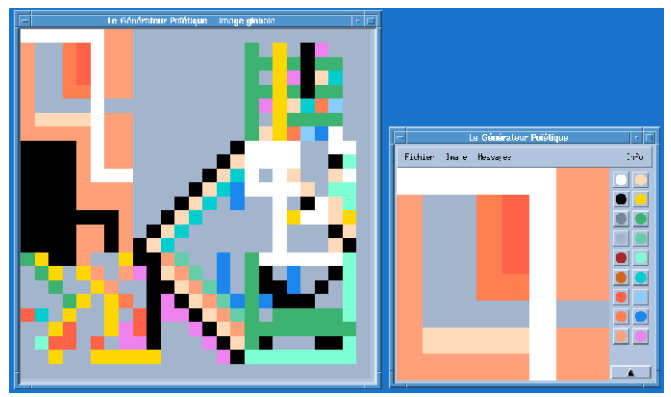

Figure 2 : le générateur poḯtique (Olivier Auber). À gauche l'image produite en temps réel par les différents contributeurs, à droite l'écran de création de l'un des contributeurs et la palette des 20 couleurs disponibles.

De nombreux artistes affectent de mettre la viralité à la question. Ainsi, Aymeric Mansoux ${ }^{32}$ invite-t-il les artistes à accepter «le fantasme» du marketing viral proposé par le web et préconise à cet effet l'adoption de règles permettant de maximiser la visibilité des œuvres au

\footnotetext{
${ }^{28}$ La vie artificielle est modélisée dans certains travaux plastiques contemporains tels Life Spacies II et Interactive plant growing des artistes Christa Sommerer et Laurent Mignonneau ce qui permet parfois en retour au scientifique de s'appuyer sur la puissance évocatrice de l'art pour donner une forme tangible aux concepts scientifique. Plusieurs plasticiens contemporains se sont d'ailleurs attachés à interroger la générativité en s'appuyant sur les potentialités du calcul informatique ou Genetic Images de Karl Sims, Massivement parallèle, Turbulence numérique, Paradis artificiels, Surnature et Nuage fractal.

${ }^{29}$ Chaque participant dessine grâce à une palette graphique rudimentaire une image de taille limitée composée de carrés « de petites ou moyenne taille» et dont on peut modifier la couleur, ce qui permet de créer des figures simples et symboliques: visages, drapeaux, bateaux... Chaque proposition constitue une partie de l'image globale qui s'affiche en temps réel pour l'ensemble des participants. La position de chaque contribution est définie d'emblée, lors de l'activation du dispositif. En cas de départ d'un participant, son dessin disparaît et la case reste blanche jusqu'à l'arrivée en ligne d'un autre inscrit. Le lecteur trouvera à l'URL http://urlz.fr/3oc4 des images animées illustrant une session du Générateur poïétique qui s'est tenue au Shanghai Institute of Visual Art le 27 novembre 2015. Les participants pouvaient alors s'installer sur l'image qu'ils généraient grâce à une application mobile et qui était projetée sur le sol.

${ }^{30}$ L'interaction proposée est collective, synchrone et « acentrée ».

${ }^{31} \mathrm{Http}: / /$ poietic-generator.net/?lang=fr_fr.

${ }^{32}$ Mcd, n69, décembre-janvier-février 2013, p. 24.
} 
sein des réseaux sociaux. Autre exemple, avec Computer Virus Joseph Nechvatal ${ }^{33}$ place au cœur de ses installations la principe même de la viralité. Cet artiste entreprend en effet de modéliser, via une programmation informatique, l'action et la prolifération de virus au sein d'un corps (ici la reproduction numérique de tableaux peints). Les différentes versions de Computer Virus vont progressivement complexifier la représentation des attaques virales. Dans la version 1 (1992), souhaitant libérer le concept de virus de toute connotation négative, l'artiste entreprend de produire des peintures à l'aide d'algorithmes mettant en jeu des processus « viraux ». Ayant par essence une forme de vie qui se déroule au sein des systèmes biologiques, les virus vont attaquer un tableau selon une action colorimétrique, créant un tableau transformé qui peut lui aussi être exposé.

À partir de 2001, l'attaque virale devient un spectacle interactif. Avec le concours de Stéphane Sikora, membre du collectif music2eye $e^{34}$ le dispositif se complexifie (Figure 3), devenant le siège d'une véritable simulation de vie artificielle dans laquelle les virus font figure d'agents autonomes, en interaction continuelle avec l'image (leur environnement). Dès lors, chaque session présente une attaque à chaque fois renouvelée et singulière correspondant à l'injection aléatoire dans l'image de différentes populations de virus. La simulation continuait jusqu'à ce qu'il ne reste plus de virus actif, donc lorsque les ressources disponibles, à savoir ici les fragments de l'image soumis à l'attaque étaient épuisées. De nouveaux cycles d'attaque pouvaient alors s'engager pour peu qu'on introduise de nouveaux virus, ayant d'autres caractéristiques...
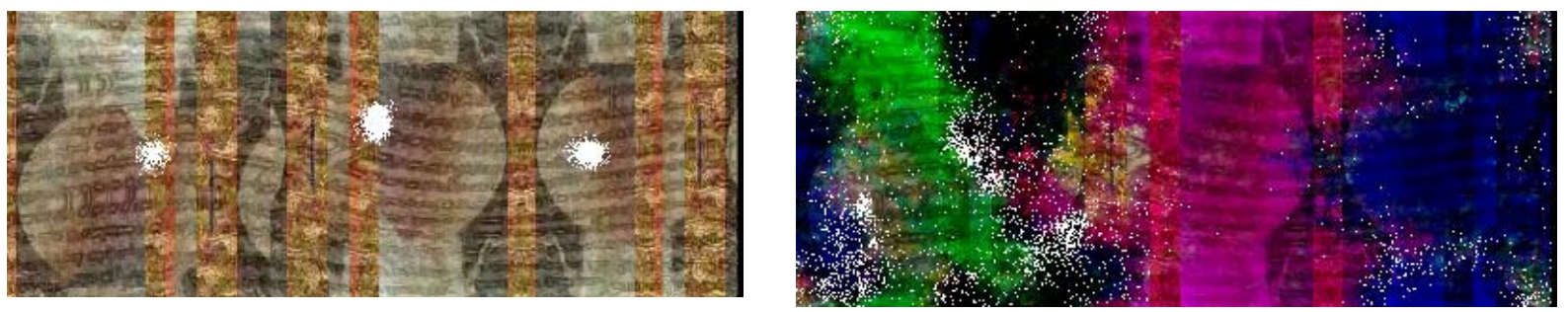

Figure 3 : Computer Virus Project, 2001-2011 (Joseph Nechvatal, Stéphane Sikora \& Music2eye).

\section{La dimension communicationnelle de la viralité questionnée par l'art}

La viralité est également questionnée dans sa dimension communicationnelle par l'art, notamment à l'instar du développement de licences open source donnant, accès sous conditions à la programmation informatique des objets numériques mis en ligne. Ainsi, promouvant l'utilisation des logiciels libres dans les champs de la musique électronique et de la création artistique, Aymeric Mansoux, artiste co-fondateur avec Thomas Vriet du collectif d'artistes GOTO10, estime-t-il que le paratexte de l'œuvre doit au même titre que l'œuvre elle-même être facilement copiable et consommé à travers les plateformes sociales. Les récits et tous les éléments contextuels participent à la transcendance de la mise en œuvre de l'installation elle-même, souvent accessible à un nombre réduit d'individus, produisant un écho qui survit à l'exercice de la performance en elle-même et implémente la mémoire collective.

Tout comme Aymeric Mansoux, Antoine Moreau soutient à travers des textes et des interventions artistiques le principe du «copyleft » à savoir la liberté pour le destinataire de diffuser - interpréter, représenter, distribuer - mais aussi de modifier des œuvres. Cette question est au cœur des valeurs fondatrices de l'internet que les enjeux d'ordre marketing ne cessent pourtant de contester. Depuis 1993, cet artiste confie des objets baptisés « sculptures » à des personnes de rencontre (Figure 4), invitant chacun à la confier par la suite à quelqu'un d'autre et ainsi de suite. Pour ces œuvres, on n'aurait donc ni propriétaire définitif ni « point

\footnotetext{
${ }^{33}$ Citons entre autres : Viral Adventures (2004), Viral symphOny (2006-2008) et Viral Venture Panel (2011). Http://www.eyewithwings.net/nechvatal/.

${ }^{34} \mathrm{Http}: / /$ www.music2eye.com/wp4/.
} 
de chute final ». L'itinérance de ces œuvres est mise en ligne par l'artiste sur un site où sont répertoriés et affichés les nom, prénom du donataire ainsi que les lieux où se sont effectuées les différentes transmissions des objets, l'identité des possesseurs successifs ainsi que la dernière localisation géographique connue de chaque «sculpture confiée ». L'internet constitue pour Antoine Moreau un moyen lui permettant de donner corps, par l'affichage de l'itinérance des œuvres, à l'idée de création open-source. Inscrivant son action dans un projet artistique militant, il contribue ainsi à porter la parole des pères fondateurs de l'Internet.

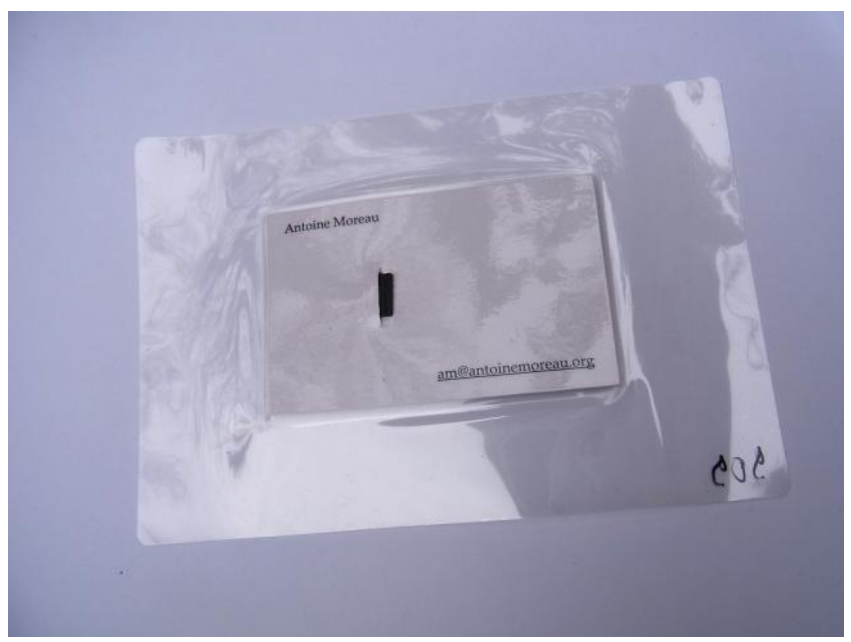

Figure 4 : sculpture confiée $n^{\circ} 909^{35}$ (Antoine Moreau, 2016).

Confronté à la virtualité développée à travers les réseaux, l'exercice de la viralité n'est pas sans risque pour la vie sociale. Avec le jeu en ligne Naked on Pluto ${ }^{36}$ (Nu sur Pluton, 2011), Aymeric Mansoux, Dave Griffiths et Marloes de Valk invitent les joueurs à prendre la mesure des méthodes intrusives en vigueur à travers l'utilisation des réseaux sociaux. La Machine à suicide Web 2.0 , existant essentiellement dans « un imaginaire nourri de vidéos en ligne », constitue pour ces artistes un appel à revenir dans le monde réel, à retrouver les liens que la virtualité des réseaux avait distendus. Cette œuvre invite l'internaute à «tuer les faux amis virtuels », à mettre définitivement fin à sa vie en ligne sans possibilité de ressusciter soimême son alter ego 2.0 chimérique afin de retrouver sa vie réelle, celle d'avant la virtualité imposée par les réseaux sociaux et, si l'on rapporte ce dispositif artistique à la propagation de l'information interrompre ou détourner l'escalade virale.

\section{Conclusion}

La viralité, avons nous vu, constitue un modèle communicationnel pertinent pour caractériser les échanges informationnels en ligne qui participent de la trivialité telle que la conceptualise Yves Jeanneret. Alternant approche communicationnelle et esthétique nous avons entrepris de déconstruire la métaphore de la viralité afin de voir comment elle se mettait en œuvre et donc comprendre le fonctionnement de la contagion informationnelle qu'elle sous entend, à savoir la propension avec laquelle les internautes bénéficient de la créativité variationnelle offerte par l'ordinateur pour produire de l'activité en ligne et en retour soigner la visibilité de leurs propos. Le développement de «mèmes numériques » sur la toile devient à ce titre, une figure majeure de l'expression de cette créativité foisonnante. La génétique nous a quant à elle

\footnotetext{
${ }^{35}$ Sculptures confiées : http://antoinemoreau.org/index.php?cat=sculptures. Lors du colloque nous avons terminé notre exposé par la présentation de la sculpture $n^{\circ} 909$ que l'artiste nous a confiée, et conformément au principe que nous lui avions proposé losque nous lui avons demandé de nous fournir une sculpture confiée, nous avons remis cet objet à une personne volontaire (madame Nada OUERGHI), acceptant de recevoir l'objet et s'engageant à le remettre en circulation et à prévenir l'artiste. http://antoinemoreau.org/index.php?art=4387.

${ }^{36}$ Voir notamment : http://fo.am/naked-on-pluto/ ainsi que le blog (http://pluto.kuri.mu) d'accompagnement.

${ }^{37} \mathrm{Http}$ ://suicidemachine.org/.
} 
permis de modéliser la contamination cellule à cellule, notamment à travers l'identification d'éléments réplicateurs.

La viralité constitue également un moteur esthétique dont s'emparent les artistes afin de donner du sens aux dialogues en ligne et de garantir la libre circulation des propositions online. Les réseaux numériques se comportent comme des «appareils » (Déotte, 2001, 2004 et Huygues, 2005) permettant d'appréhender la complexité contemporaine. La multiplication et la diversité des propositions artistiques confèrent à l'image actée (Barboza, Weissberg, 2006) de nouveaux statuts. À l'ère de la mobilité et de la connectivité généralisées, l'image constitue en effet une accroche et un vecteur conversationnel majeur. Saisie par l'intensification des échanges qui se développent en ligne, elle est à la fois objet et sujet des contagions informationnelles qui marquent la toile. Rien d'étonnant que certains annonceurs privilégient désormais le recours à Instagram et Snapchat $^{38}$, dispositifs relationnels plus à même de développer le picture marketings. Il s'agit par ce biais de dégager à travers les logiques d'actions (Beauvisage et al, 2011: 161) des réseaux sociaux en général les lignes forces propres à Instagram et Snapchat, et prendre élan sur l'augmentation du rapport de proximité que renforcent ces réseaux sociaux, mieux adaptés à la connexion nomade que Facebook et Twitter, réseaux sociaux dominants, afin d'élaborer un nouveau rapport à l'objet dont on veut faire la publicité. Tissant des liens directs avec les internautes, la publicité devient conversationnelle et narrative, articulant en temps réel les témoignages visuels liés au produit dont on veut assurer la promotion.

\section{Références}

Beauvisage T., Beuscart J-S., Thomas Couronné T. \& Mellet K., 2011, "Le succès sur Internet repose-t-il sur la contagion ? Une analyse des recherches sur la viralité ». Tracés. Revue de Sciences humaines, 21, 151-166. DOI : 10.4000/traces.5194. Accès : http://traces.revues.org.bases-doc.univ-lorraine.fr/5194 (dernière consultation le 19 mai 2016)

Barboza P., Weissberg J.-L., dirs., 2006, L'image actée. Scénarisations numériques, parcours du séminaire. L'action sur l'image. Paris, Éd. L'Harmattan, coll. Champs visuels.

Bonenfant M., 2014, «Le mème numérique : étude sémiotique des réseaux à partir des concepts de trace et d'indice ». RISCP, 2014/12. DOI : 10.4000/communiquer.1295.

Breton P., 2000, Le culte d'internet. Une menace pour le lien social. Paris, Éd. La Découverte.

Couchot É., 2007, Des images, du temps et des machines dans les arts et la communication, Nîmes, éd. Jacqueline Chambon.

Dawkins C. R., 1996, Le gène égoïste. (2e éd.). Trad. de l'anglais par Laura Ovion. Paris, Éd. Odile Jacob.

De Certeau M., 1980, L'Invention du quotidien. 1. Arts de faire, Paris, Gallimard, 1990.

De Kerckhove D., 1997, L'intelligence des réseaux, Paris, O. Jacob.

De Kerkhove D., 1999, « Penser à l'écran », Art Press hors série. L'art et la toile, pp. 80-88.

Déotte J.-L., 2001, L'époque de l'appareil perspectif. Brunelleschi, Machiavel, Descartes, Paris, L'Harmattan.

Déotte J.-L., 2004, L'époque des appareils, Paris, Éd. Lignes/Manifeste, Léo Scheer.

\footnotetext{
${ }^{38}$ En Août 2015, Instagram comptait 400 millions d'utilisateurs actifs et Snapchat 100 millions. Ces deux platesformes qui basent leurs stratégies sur les contenus visuels sont, semble t-il, en pleine expansion, notamment dans les pratiques communicationnelles des adolescents. http:/www.blogdumoderateur.com/chiffres-reseaux-sociaux/. Consulté le 20/10/2016.

${ }^{39}$ Tendance émergente qui s'observe sur les médias sociaux depuis plusieurs années et qui prend acte de l'importance, pour les entreprises et les marques de l'image, contenu rapidement identifiable par les internautes et facilement partageable. Cf. http://www.webmarketing-com.com/2014/03/17/26547-le-picture-marketingcreer-de-la-valeur-par-limage. Consulté le 20/10/2016.
} 
Huyghe P.-D. dir., 2005, L'art au temps des appareils, Paris, L'Harmattan.

Flichy P., 1995, L'innovation technique. Récents développements en sciences sociales. Vers une nouvelle théorie de l'innovation. Paris, Éd. La découverte.

Granier J.-M., 2001, « Du Contrat de lecture au Contrat de conversation ». Communication et langages, 100, pp. 51-62.

Gunthert A., 2009, «L'image partagée : Comment internet a changé l'économie des images », Études photographiques, 24, pp. 182-209. Accès: http://etudesphotographiques.revues.org/2832. Consulté le 20/10/1016.

Gunthert A., 2011, «L'œuvre d'art à l'ère de son appropriabilité numérique ». Les Carnets du $B A L, 2$, 136-149. Accès : http://culturevisuelle.org/icones/2191. Consulté le 20/10/1016.

Jakobson R., 1963, Essais de linguistique générale, Paris, Éd. de Minuit.

Jeanneret Y., 2008, Penser la trivialité. Volume 1. La vie triviale des êtres culturels, Paris, Éd. Hermes-Lavoisier.

Jouët J., 2000, Retour critique sur la sociologie des usages. Réseaux, 18, 100, pp. 487-521.

Moles A., 1990, Art et ordinateur. Paris, Blusson.

Morelli P., 2000, Multimédia et création. Contribution des artistes au développement d'une écriture multimédia, Thèse de doctorat en sciences de l'information et de la communication, université de Metz. Accès : http://docnum.univ-lorraine.fr/public/UPVM/Theses/2000/Morelli.Pierre.LMZ0012_1.pdf. Et $\quad$ Et $\quad$ http:/docnum.univlorraine.fr/public/UPV-M/Theses/2000/Morelli.Pierre.LMZ0012_2.pdf.

Morelli P., 2016, «Les réseaux numériques mis à la question par l'art. Interrogations esthétiques et plastiques des technologies émergentes ». Revue Essais, Réseau(x). Bordeaux. À paraître.

Proulx S., 2005, « Penser les usages des technologies de l'information et de la communication aujourd'hui : enjeux, modèles, tendances », pp. 15-17, in Vieira L., Pinède-Wojciechowski N., dirs, Enjeux et usages des TIC : aspects sociaux et culturels. Tome 1, Bordeaux, Presses universitaires de Bordeaux.

Schlanger J., 1983, L'invention intellectuelle, Paris, Fayard.

Tisseron S., 2011, «Intimité et extimité », Communications, 88, p. 83-91.

Virilio P., 1988, « La fin des certitudes », Entretien avec Fred Forest, Art Press, 122, p. 14. 